\begin{tabular}{|c|c|c|c|l|l|}
\hline \multirow{2}{*}{$\mathcal{O}$} & J O O R & \multicolumn{1}{|c|}{$\mathbf{1 2 3 6 9}$} & \multirow{2}{*}{ WILEY } & Dispatch: 30.10 .15 & CE: Vijay T \\
\cline { 2 - 3 } \cline { 5 - 6 } & Journal Code & Manuscript No. & & No. of pages: 7 & PE: Mohanapriya \\
\hline
\end{tabular}

\title{
Peripheral, functional and postural asymmetries related to the preferred chewing side in adults with natural dentition
}

\author{
B. ROVIRA-LASTRA* ${ }^{\dagger}$, E. I. FLORES-OROZCO**, R. AYUSO-MONTERO* ${ }^{\dagger}$,
}

M. PERAIRE $*^{\dagger} \&$ J. MARTINEZ-GOMIS $*^{\dagger}$ *Department of Prosthodontics, Faculty of Dentistry, University of Barcelona, Barcelona, 'Oral Health and Masticatory System Group (Bellvitge Biomedical Research Institute) IDIBELL, L'Hospitalet de

3 Llobregat,Barcelona, Spain and "Department of Prosthodontics, Faculty of Dentistry, Autonomous University of Nayarit, Nayarit, Mexico

\begin{abstract}
SUMMARY The aim of this cross-sectional study was to determine the preferred chewing side and whether chewing side preference is related to peripheral, functional or postural lateral preferences. One hundred and forty-six adults with natural dentition performed three masticatory assays, each consisting of five trials of chewing three pieces of silicon placed into a latex bag for 20 cycles, either freestyle or unilaterally on the right- or left-hand side. Occlusal contact area in the intercuspal position, maximum bite force, masticatory performance and cycle duration were measured and the lateral asymmetry of these variables was calculated. Laterality tests were performed to determine handedness, footedness, earedness and eyedness as functional preferences, and hand-clasping, arm-folding and leg-crossing as postural lateral preferences. The preferred chewing side was determined using three different methods: assessment of the first chewing cycle for each trial, calculation of the asymmetry index
\end{abstract}

from all cycles and application of a visual analogue scale. Bivariate relationship and multiple linear regression analyses were performed. Among unilateral chewers, $77 \%$ of them preferred the right side for chewing. The factors most closely related to the preferred chewing side were asymmetry of bite force, asymmetry of masticatory performance and earedness, which explained up to $16 \%$ of the variance. Although several functional or postural lateral preferences seem to be related to the preferred chewing side, peripheral factors such as asymmetry of bite force and of masticatory performance are the most closely related to the preferred chewing side in adults with natural dentition.

KEYWORDS: cross-sectional studies, functional laterality, humans, lateral preference, mastication, regression analysis

Accepted for publication 12 October 2015

\section{Introduction}

One of the main objectives of prosthodontic treatment is to restore or improve masticatory function. Although the restoration of missing posterior teeth on the preferred chewing side (PCS) would improve masticatory efficiency, it remains to be determined whether prosthodontic restoration on the non-preferred side would change the masticatory pattern and thus improve masticatory performance $(1,2)$. This depends on whether the selection of the chewing side is regulated primarily by peripheral asymmetries or is an expression of cerebral dominance, like other functional lateralities. Peripheral factors that have been found to be related to masticatory laterality include asymmetry of several characteristics of occlusion, bite force and masticatory performance, missing posterior teeth and temporomandibular disorders (3-7), most of them are interrelated $(4,7)$. However, other studies have failed to find a significant association between some of these peripheral factors and the PCS $(2,8-10)$.

Functional preferences such as handedness, footedness, earedness and eyedness are thought to be related to cerebral dominance and have been found 
to be significantly and positively correlated with masticatory laterality in some studies $(2,11)$, but not in others $(4,8,12)$. Other lateralities are postural preferences such as hand-clasping, arm-folding and legcrossing preferences, which are affected by cultural factors to a lesser degree than handedness (13). Legcrossing was associated with handedness, footedness, earedness and eyedness (13). Moreover, the strength of preference for arm-folding and hand-clasping was correlated (14), and handedness was associated with both postural preferences (15). To our knowledge, no studies have been performed to determine the relationship between masticatory laterality and postural lateral preferences. If these lateral preferences are related, it would suggest that they are controlled by the same factors.

The percentage of children and adults with a PCS ranges from $45 \%$ to $98 \%$ and there is no agreement on the question of which side is used more frequently $(2-4,8,11,16)$. Discrepancies in the literature can be explained by differences in study populations and the definition of PCS, and consequently the methods used, including the type of test food used, the measurement technique employed and the number of cycles assessed (17). Among the objective methods, the use of bagged silicone to assess either the first cycle or all cycles showed a high level of reproducibility and validity (17). Although it is not an objective method, the use of the VAS to describe the level of lateral preference in mastication perceived by the subject was shown to be a valid and reliable method for the quantitative assessment of the PCS (17).

The main objective of this study was to determine whether peripheral, functional or postural lateralities are related to the preferred chewing side assessed by three different methods in adults with natural dentition. This study also aimed to determine if the right side was used more frequently for chewing among the same population. The null hypothesis tested was that the right side is used as frequently for chewing as the left side.

\section{Material and methods}

Subjects

In this cross-sectional study, 146 young adults (102 women and 44 men, age range $20-45$ years, mean age 24.4) with natural dentition were recruited from volunteer students and staff at the Faculty of Dentistry at the University of Barcelona (Barcelona, Spain). Among the participants, 42 had participated in an earlier research project $(7,18)$. Subjects with fewer than 24 natural teeth, those undergoing active orthodontic treatment and those suffering from orofacial pain were excluded. No subject had severe malocclusion or temporomandibular disorders that could affect mandibular movement. A test-retest was performed in 10 subjects, chosen by convenience, 1-2 weeks after the first measurements. Subjects were fully informed and signed an informed consent form approved by Barcelona University Dental Hospital Ethics Committee (Code 06/14). All experiments were carried out in accordance with the principles of the Helsinki Declaration.

\section{Laterality tests}

A single researcher performed all laterality tests. Handedness was determined by observing which hand the participant used to stir liquid in a glass. Footedness was assessed by observing which foot was used to stamp on a paper placed on the floor. Earedness was determined by observing which ear was used for listening through a hole. Eyedness was assessed by observing which eye was used for looking into a dark bottle (2). Hand-clasping, arm-folding and leg-crossing were determined by observing which thumb, arm or leg was positioned uppermost when the participants clasped their hands, folded their arms or crossed their legs, respectively (13). Preference for the right was coded as +1 , whereas preference for the left was coded as -1 .

\section{Bite force and occlusal contact area}

A bite-force transducer (gnathodynamometer*) was used to measure unilateral maximum bite force between the first premolars on both sides (4). The bite force measurements were recorded three times, with the order changed for each test, and the highest value was selected for each side. Occlusal contact area in the intercuspal position was measured on the left and right sides using bite registration material (Occlufast Rock $^{\dagger}$ ) (4). Occlusal registrations were obtained from

*Technical University of Catalonia, Barcelona, Spain.

†Zhermack, Badia Polesine, Italy. 
all subjects, trimmed, scanned and analysed by means of computer software (UTHSCSA Image Tool V3.0 $0^{\ddagger}$ ). Occlusal contact was considered as an interocclusal distance of $200 \mu \mathrm{m}$ or less, because it was found to be the most correlated with masticatory performance (19).

\section{Masticatory assays}

Each subject performed three masticatory assays, each consisting of five trials of chewing $2 \mathrm{~g}$ of silicon for 20 cycles. Optosil tablets $(5 \mathrm{~mm}$ thick, $20 \mathrm{~mm}$ in diameter) (Optosil P Plus ${ }^{\S}$ ) were made as described by Albert et al. (20), cut into quarters and three of the quarter tablets were placed in a latex bag, which was then sealed (7). In order to assess the PCS, one assay consisted of freestyle mastication. In order to assess masticatory performance and chewing rate, in the other two assays, subjects were asked to chew bagged silicon unilaterally, i.e. only on the right-hand side in one assay and only on the left-hand side in the other, and the order of these trials was alternated.

Masticatory performance was evaluated for each unilateral masticatory assay by assessing the degree of comminution of the silicon test food (19). For each assay, particles from five trials ( $10 \mathrm{~g}$ ) were dried for $24 \mathrm{~h}$ and passed through a series of eight sieves $(0.25,0.425$, $0 \cdot 85,2,2 \cdot 8,3 \cdot 15,4$, and $5.6 \mathrm{~mm}$ ) while being shaken for $1 \mathrm{~min}$. After the cumulative weight distribution of the sieve contents had been determined, the median particle size was calculated for each subject using the Rosin-Rammler equation [Qw $\left.(\mathrm{X})=1-2 \mathrm{E}-\left(\mathrm{X} / \mathrm{X}_{50}\right)^{\mathrm{b}}\right]$; where $\mathrm{Qw}(\mathrm{X})$ is the fraction of particles by weight with a diameter smaller than $X$, the median particle size (MPS or $\mathrm{X}_{50}$ ) is the size of a theoretical sieve through which $50 \%$ of the weight can pass and $b$ describes the breadth of particle size distribution (21). The total duration of each of the five trials was used to calculate the duration of the average chewing cycle for each unilateral masticatory assay (22).

\section{Methods for determining the PCS}

The PCS was determined by three methods. The first method (PCS_l ${ }^{\text {st }} \mathrm{C}$ ) was based on the definition of

tUniversity of Texas Health Science Center, San Antonio, TX, USA. ${ }^{\S}$ Heraeus Kulzer, Hanau, Germany.
PCS as "the mandibular side favoured during the closing phase for the first masticatory cycle" (12), measured five times using an index as follows: $\mathrm{I}=$ (right - left)/(right + left). The second method (PCS_AI) used all masticatory cycles to calculate the asymmetry index (AI), as follows: $\mathrm{AI}=$ (number of right strokes - number of left strokes)/(number of right strokes + number of left strokes) (23). The last method (PCS_VAS) consisted of using a VAS assessment in which subjects made a mark on a $10-\mathrm{cm}$ line with 'always left' (-1) and 'always right' (+1) at either end and 'no preference' (0) in the middle (17).

\section{Data analysis}

New variables called 'Asymmetry of...' were calculated for each peripheral variable parameter as the absolute difference between right-hand side values and left-hand side values. Therefore, a positive value for an asymmetry of quantitative variables signifies that the right-side value is higher than that of the left side. The PCS values of each subject in each of the three methods range from -1 (extreme left-side chewer) to +1 (extreme right-side chewer). To determine whether each subject had a chewing side preference, the threshold was set at 33\%; so that the subject was considered an alternate unilateral chewer if the AI value for PCS ranged from -0.33 to 0.33 and a consistent unilateral chewer if the AI value was less than -0.33 or more than 0.33 . This threshold was selected in order to obtain three balanced groups and according to other studies $(7,23)$. Intra-class correlation coefficients and kappa statistics were determined as measures of the reliability of quantitative and categorical variables, respectively (Table 1). Quantitative variables showed ICC values higher than $0 \cdot 6$, indicating a high discrimination between participants and high agreement between sessions. All quantitative variables, except for PCS_l ${ }^{\text {st }} \mathrm{C}$, were normally distributed $(P>0 \cdot 05$, Kolmogorov-Smirnov). Pearson or Spearman's Rho correlation coefficients were calculated to evaluate the correlation between peripheral, functional or postural variables, and masticatory laterality was determined for each method. Finally, since the variables were interrelated, a stepwise multiple linear regression analysis with an inclusion level of 0.05 was performed to examine whether the variables would significantly contribute to explaining the preferred chewing side determined for each method. 
Table 1. Reproducibility of the variables estimated using intraclass correlation coefficients (ICC) and kappa statistics with 95\% confidence interval $(\mathrm{CI})$ coefficients $(n=10)$

\begin{tabular}{ll}
\hline Variables & ICC $(95 \%$ CI $)$ \\
\hline PCS_l ${ }^{\text {st } C}$ & $0.79(0.40: 0.94)$ \\
PCS_AI & $0.77(0.37: 0.93)$ \\
PCS_VAS & $0.93(0.77: 0.98)$ \\
Asym_MPS (mm) & $0.81(0.22: 0.95)$ \\
Asym_TIME (ms) & $0.82(0 \cdot 27: 0.96)$ \\
Asym_BF $(\mathrm{N})$ & $0.68(0 \cdot 11: 0.91)$ \\
Asym_OCA $\left(\mathrm{mm}^{2}\right)$ & $0.69(0 \cdot 16: 0.91)$ \\
\hline Variables & Kappa $(95 \% \mathrm{CI})$ \\
\hline Handedness & $0.82(0.42: 0.95)$ \\
Footedness & 1 \\
Earedness & $0.82(0.42: 0.95)$ \\
Eyedness & 1 \\
Hand-clasping & 1 \\
Arm-folding & 1 \\
Leg-crossing & $0.82(0.42: 0.95)$ \\
\hline
\end{tabular}

PCS_l ${ }^{\text {st }} \mathrm{C}=$ Preferred chewing side based on the lst cycle. PCS_AI $=$ Preferred chewing side based on the asymmetry index. PCS_VAS $=$ Preferred chewing side based on the visual analogue scale. Asym = asymmetry; MPS = median particle size; TIME $=$ cycle duration; $\mathrm{BF}=$ bite force; $\mathrm{OCA}=$ occlusal contact area.

Statistical analysis was performed using the SPSS programme (IBM SPSS Statistics, version 21.0\%) and $P$-values below 0.05 were considered significant.

\section{Results}

Regardless of the method used to determine the PCS, the subjects tended to chew more frequently on the right-hand side as demonstrated by the $95 \%$ confidence interval that did not include a mean of 0 and was positive (Table 2). Using the threshold of $33 \%$, $56 \%$ of the participants were classified as consistent unilateral chewers (23\% left-hand side and $77 \%$ right-hand side) and $44 \%$ as alternate unilateral chewers. For each functional laterality, the right side was preferred over the left side, handedness (93\%), footedness $(95 \%)$, earedness $(71 \%)$ and eyedness $(71 \%)$. However, like postural preferences, the left thumb was used more frequently than the right thumb while clasping the hands $(64 \%)$, the right leg was used more frequently than the left leg while

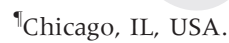

Table 2. Descriptive analysis of the preferred chewing side, peripheral asymmetries and functional and postural lateralities of the subjects $(n=146)$

\begin{tabular}{|c|c|c|c|c|}
\hline & Mean & CI $95 \%$ of mean & $\begin{array}{l}\text { Right } \\
\%\end{array}$ & $\begin{array}{l}\text { Left } \\
\%\end{array}$ \\
\hline PCS_l ${ }^{\text {st }} C^{*}$ & $0 \cdot 60$ & $(-0.20$ and 1.0$)$ & 45.5 & $16 \cdot 4$ \\
\hline PCS_AI & $0 \cdot 22$ & $(0 \cdot 14$ to $0 \cdot 29)$ & $34 \cdot 9$ & $10 \cdot 3$ \\
\hline PCS_VAS & 0.29 & $(0.22$ to 0.37$)$ & 45.5 & $14 \cdot 4$ \\
\hline Asym_MPS (mm) & 0.08 & $(-0.06$ to 0.22$)$ & & \\
\hline Asym_TIME (ms) & $0 \cdot 45$ & $(-3 \cdot 4$ to $4 \cdot 3)$ & & \\
\hline Asym_BF (N) & 1.82 & $(0.65$ to 3.0$)$ & & \\
\hline Asym_OCA $\left(\mathrm{mm}^{2}\right)$ & $-1 \cdot 15$ & $(-3 \cdot 1$ to $0 \cdot 8)$ & & \\
\hline Handedness & $0 \cdot 85$ & $(0 \cdot 76$ to 0.94$)$ & $92 \cdot 5$ & $7 \cdot 5$ \\
\hline Footedness & $0 \cdot 89$ & $(0.82$ to 0.97$)$ & $94 \cdot 5$ & 5.5 \\
\hline Earedness & $0 \cdot 41$ & $(0 \cdot 26$ to $0 \cdot 56)$ & $70 \cdot 5$ & $29 \cdot 5$ \\
\hline Eyedness & 0.41 & $(0 \cdot 26$ to 0.56$)$ & $70 \cdot 5$ & $29 \cdot 5$ \\
\hline Hand-clasping & $-0 \cdot 29$ & $(-0.44$ to -0.13$)$ & $35 \cdot 6$ & $64 \cdot 4$ \\
\hline Arm-folding & $-0 \cdot 14$ & $(-0 \cdot 30$ to $0 \cdot 03)$ & $43 \cdot 2$ & $56 \cdot 8$ \\
\hline Leg-crossing & $0 \cdot 64$ & $(0.52$ to $0 \cdot 77)$ & $82 \cdot 2$ & $17 \cdot 8$ \\
\hline
\end{tabular}

$\mathrm{CI}=$ confidence interval; PCS_l ${ }^{\text {st }} \mathrm{C}=$ Preferred chewing side based on the 1st cycle. PCS_AI = Preferred chewing side based on the asymmetry index. PCS_VAS = Preferred chewing side based on the visual analogue scale. Asym = asymmetry; MPS = median particle size; TIME $=$ cycle duration; $\mathrm{BF}=$ bite force; $\mathrm{OCA}=$ occlusal contact area.

*Because this variable was not distributed normally, these values refer to median and percentile 25 and 75 .

crossing the legs $(82 \%)$ and no lateral predominance was observed in arm-folding.

The Pearson correlation coefficients between the PCS determined for each method and the laterality of peripheral, functional and postural variables are shown in Table 3. Asymmetry of bite force, asymmetry of masticatory performance and earedness significantly correlated with PCS, regardless of the method used to determine it. In other words, the right chewers tended to have higher bite force on the right side, higher masticatory performance (lower MPS) on the right side and used more frequently the right ear. Asymmetry of occlusal contact area and leg-crossing were weakly related to PCS and were dependent on the method used.

Stepwise multiple regression analysis showed that asymmetry of bite force and asymmetry of masticatory performance were the most important factors affecting the PCS determined using the asymmetry index (Table 4). These two variables accounted for $16 \%$ of the variation in PCS (adjusted $R^{2}=0 \cdot 16$ ). However, earedness, asymmetry of bite force and 
Table 3. Correlation coefficient between the preferred chewing side (PCS) and peripheral asymmetries and functional and postural lateralities

\begin{tabular}{|c|c|c|c|}
\hline & $\begin{array}{l}\text { PCS_1st Cycle } \\
\text { (Rho } \\
\text { Spearman) }\end{array}$ & $\begin{array}{l}\text { PCS_Asymmetry } \\
\text { Index (Pearson's } \\
\text { r) }\end{array}$ & $\begin{array}{l}\text { PCS_VAS } \\
\text { (Pearson's } \\
\text { r) }\end{array}$ \\
\hline Asym__MPS & $-0 \cdot 24^{* *}$ & $-0.27 * *$ & $-0.23 * *$ \\
\hline Asym__Time & -0.04 & -0.07 & -0.07 \\
\hline Asym__BF & $0.33 * *$ & $0.34^{* *}$ & $0 \cdot 24^{* *}$ \\
\hline Asym_OCA & $0 \cdot 04$ & $0 \cdot 12$ & $0 \cdot 17^{*}$ \\
\hline Handedness & 0.05 & 0.02 & 0.03 \\
\hline Footedness & 0.02 & 0.08 & 0.08 \\
\hline Earedness & $0 \cdot 19 *$ & $0 \cdot 20^{*}$ & $0 \cdot 25^{* *}$ \\
\hline Eyedness & $0 \cdot 10$ & -0.03 & -0.06 \\
\hline $\begin{array}{l}\text { Hand- } \\
\text { clasping }\end{array}$ & $0 \cdot 04$ & 0.08 & 0.07 \\
\hline Arm-folding & -0.08 & $-0 \cdot 02$ & $-0 \cdot 04$ \\
\hline Leg-crossing & $0 \cdot 10$ & $0 \cdot 18^{*}$ & $0 \cdot 22 * *$ \\
\hline
\end{tabular}

$* P<0.05 ; * * P<0.01 ;$ PCS $=$ preferred chewing side; Asym $=$ asymmetry; MPS = median particle size; TIME = cycle duration; $\mathrm{BF}=$ bite force; $\mathrm{OCA}=$ occlusal contact area. Bold values mean significant correlation.

Table 4. Stepwise regression models of factors related to the preferred chewing side (PCS) determined using the asymmetry index and the visual analogue scale (VAS)

\begin{tabular}{|c|c|c|c|c|c|}
\hline Model & Variables included & Beta & $R$ & $R_{a}^{2}$ & $F$ (Sig.) \\
\hline \multicolumn{6}{|c|}{ PCS-Asymmetry index } \\
\hline 1 & $\begin{array}{l}\text { Asymmetry of bite } \\
\text { force }(\mathrm{N})\end{array}$ & $0 \cdot 34$ & $0 \cdot 34$ & $0 \cdot 11$ & $19.0(<0.001)$ \\
\hline 2 & $\begin{array}{l}\text { Asymmetry of } \\
\text { masticatory } \\
\text { performance } \\
(\mathrm{mm})\end{array}$ & -0.23 & 0.41 & $0 \cdot 16$ & $14.4(<0.001)$ \\
\hline \multicolumn{6}{|c|}{ PCS-VAS } \\
\hline 1 & Earedness & $0 \cdot 25$ & $0 \cdot 25$ & $0 \cdot 06$ & $9.4(0.003)$ \\
\hline 2 & $\begin{array}{l}\text { Asymmetry of bite } \\
\text { force }(\mathrm{N})\end{array}$ & $0 \cdot 20$ & $0 \cdot 32$ & $0 \cdot 09$ & $8.0(0.001)$ \\
\hline 3 & Leg-crossing & $0 \cdot 19$ & 0.37 & $0 \cdot 12$ & $7.4(<0.001)$ \\
\hline
\end{tabular}

$R_{a}^{2}$ : adjusted $R^{2}$ (fraction of variance explained). F (Sig.): $F$-value and significance.

leg-crossing accounted for $12 \%$ of the variation in the PCS perceived by the subject through a VAS.

\section{Discussion}

Among all the variables studied, asymmetry of bite force, asymmetry of masticatory performance and earedness were shown to be the factors most closely related to the PCS, since they explained up to $16 \%$ of the variance in adults with natural dentition. This study also confirmed that the right side is more frequently preferred for chewing than the left side, and therefore, the null hypothesis was rejected. These two findings support the fact that although the PCS is influenced by a central mechanism $(2,11)$, masticatory laterality depends more on peripheral asymmetries, especially those related to masticatory performance, when chewing hard food $(3,4,7,12)$.

These results suggest that only $16 \%$ of the PCS variation can be attributed to peripheral or central factors. Therefore, $84 \%$ of this variation is caused by other variables that were not included in this study. It has been reported that a complex interplay of factors is associated with the PCS, and therefore the side chosen for chewing in most cycles may occur at random $(8,24)$. The population studied consisted of young adults with natural dentition and without pain or dental caries, so it is reasonable to assume that these subjects were able to masticate on either side and preferred to alternate between sides. By contrast, a study that was conducted with a more heterogeneous population found that $63 \%$ of the variation in unilateral or bilateral chewing could be explained by gender and age, and several peripheral factors such as temporomandibular symptoms, asymmetric tooth loss and use of a removable prosthetic restoration (3). In the same study, the preferred chewing side was significantly associated with unilateral temporomandibular joint pain, unilateral joint clicking and asymmetric tooth loss (3).

The percentage of right-sided subjects for functional and postural lateralities found in this study is consistent with that reported in another study (13). The fact that there is a significant preference for chewing on the right side over the left side has also been reported in other studies conducted among different populations $(2-4,11,16)$. However, the correlation between masticatory laterality and handedness was found to be significant in just a few studies $(2,11)$, but not in others $(4,8,10)$. These discrepancies may be explained by the population studied and the method used to determine the PCS. It is possible that the type of test food used may influence the PCS, since studies that used hard food, such as silicone or carrots, failed to find a correlation between masticatory laterality and handedness $(4,8,10)$ in contrast to studies that used chewing gum $(2,11)$. People usually chew gum for fun and the process may be more centrally controlled, whereas the aim of chewing hard food is to 
break the food down, and this probably requires use of the most efficient side for chewing.

Among the functional lateralities, earedness was the most closely related to masticatory laterality, as has been reported in other studies $(2,11,12)$. In fact, earedness is apparently a better predictor than handedness, footedness or eyedness of hemispheric predominance for language (25). Another possible explanation is that the proportion of right-side chewers is more similar to the proportion of right-earedness than right-handedness or right-footedness. Similarly, the significant relationship between legcrossing and chewing laterality, although weak, may be influenced by the similarity of the proportions. Therefore, it seems plausible that in absence of relevant peripheral asymmetries, the central nervous system controls the side for chewing.

This study confirms that asymmetry of peripheral factors are more directly correlated with masticatory laterality than central factors. Consequently, it is possible that restoration of missing posterior teeth on the non-preferred side would change masticatory laterality and improve masticatory performance. However, due to the cross-sectional design of this study, we cannot demonstrate whether an increase in bite force and masticatory performance on one side is the result or the cause of the preference of this side for chewing. Therefore, confirmation of this hypothesis is required through a prospective analytical study. Another limitation of this study is that, due to the low percentage of subjects with left-sided functional lateralities, the sample size is small and this limits the ability to detect weaker associations. Furthermore, only one task for each functional laterality was evaluated and we cannot rule out associations with other functional laterality aspects. Another of the study's weaknesses was its use of only one test food to assess the PCS. The results are therefore only applicable to this type of test food.

In conclusion, although several functional or postural lateral preferences seem to be related to the preferred chewing side, peripheral factors such as asymmetry of both bite force and masticatory performance are the most closely related to the preferred chewing side in adults with natural dentition.

\section{Acknowledgments}

The authors thank Christopher Evans for correcting their English. This study was approved by Barcelona
University Dental Hospital Ethics Committee (Code 06/14).

\section{Funding}

This research was partially funded by the Bellvitge Campus Research Committee, University of Barcelona (ACESB 08/04), and the Faculty of Dentistry, University of Barcelona.

\section{Conflicts of interest}

The authors declare that there are no conflicts of interest.

\section{References}

1. van der Bilt A, Olthoff LW, Bosman F, Oosterhaven SP. Chewing performance before and after rehabilitation of post-canine teeth in man. J Dent Res. 1994;73:1677-1683.

2. Nissan J, Berman O, Gross O, Haim B, Chaushu G. The influence of partial implant-supported restorations on chewing side preference. J Oral Rehabil. 2011;38:165-169.

3. Diernberger S, Bernhardt O, Schwahn C, Kordass B. Selfreported chewing side preference and its associations with occlusal, temporomandibular and prosthodontic factors: results from the population-based Study of Health in Pomerania (SHIP-0). J Oral Rehabil. 2008;35:613-620.

4. Martinez-Gomis J, Lujan-Climent M, Palau S, Bizar J, Salsench J, Peraire M. Relationship between chewing side preference and handedness and lateral asymmetry of peripheral factors. Arch Oral Biol. 2009;54:101-107.

5. Lamontagne P, Al-Tarakemah Y, Honkala E. Relationship between the preferred chewing side and the angulation of anterior tooth guidance. Med Princ Pract. 2013;22:545-549.

6. Tomonari H, Kubota T, Yagi T, Kuninori T, Kitashima F, Uehara $\mathrm{S}$ et al. Posterior scissors-bite: masticatory jaw movement and muscle activity. J Oral Rehabil. 2014;41:257-265.

7. Rovira-Lastra B, Flores-Orozco EI, Salsench J, Peraire M, Martinez-Gomis J. Is the side with the best masticatory performance selected for chewing? Arch Oral Biol. 2014;59:1316-1320.

8. Pond LH, Barghi N, Barnwell GM. Occlusion and chewing side preference. J Prosthet Dent. 1986;55:498-500.

9. Wilding RJ, Adams LP, Lewin A. Absence of association between a preferred chewing side and its area of functional occlusal contact in the human dentition. Arch Oral Biol. 1992;37:423-428.

10. Salioni MA, Pellizoni SE, Guimarães AS, Juliano Y, Alonso LG. Functional unilateral posterior crossbite effects on mastication movements using axiography. Angle Orthod. 2005;75:362-367.

11. Barcellos DC, da Silva MA, Batista GR, Pleffken PR, Pucci $\mathrm{CR}$, Borges $\mathrm{AB}$ et al. Absence or weak correlation between chewing side preference and lateralities in primary, mixed 
and permanent dentition. Arch Oral Biol. 2012;57:10861092.

12. Hoogmartens MJ, Caubergh MA. Chewing side preference in man correlated with handedness, footedness, eyedness and eardness. Electromyogr Clin Neurophysiol. 1987;27:293-300.

13. Dittmar M. Functional and postural lateral preferences in humans: interrelations and life-span age differences. Hum Biol. 2002;74:569-585.

14. Ogah I, Stewart E, Treleaven M, Wassersug RJ. Hand clasping, arm folding, and handedness: relationships and strengths of preference. Laterality. 2012;17:169-179.

15. Tran US, Koller I, Nader IW, Pietschnig J, Schild AH, Stieger $\mathrm{S}$ et al. Lateral preferences for hand clasping and arm folding are associated with handedness in two large-sample latent variable analyses. Laterality. 2014;19:602-614.

16. Paphangkorakit J, Thothongkam N, Supanont N. Chewingside determination of three food textures. J Oral Rehabil. 2006;33:2-7.

17. Flores-Orozco EI, Rovira-Lastra B, Peraire M, Salsench J, Martinez-Gomis J. The reliability of a visual analog scale for determining the preferred hering side. J Prosthet Dent. in press. 2015;??????????-????. DOI: 10.1016/j.prosdent. $5 \quad 2015.07 .006$

18. Flores-Orozco EI, Rovira-Lastra B, Willaert E, Peraire M, Martinez-Gomis J. Relationship between jaw movement and masticatory performance in adults with natural dentition. Acta Odontol Scand. in press. 2015;????:????-???. DOI: $10.3109 / 00016357.2015 .1048996$
19. Lujan-Climent M, Martinez-Gomis J, Palau S, Ayuso-Montero $R$, Salsench $J$, Peraire $M$. Influence of static and dynamic occlusal characteristics and muscle force on masticatory performance in dentate adults. Eur J Oral Sci. 2008; 116:229-236.

20. Albert TE, Buschang PH, Throckmorton GS. Masticatory performance: a protocol for standardized production of an artificial test food. J Oral Rehabil. 2003:30:720-722.

21. Olthoff LW, van der Bilt A, Bosman F, Kleizen HH. Distribution of particle sizes in food comminuted by human mastication. Arch Oral Biol. 1984;29:899-903.

22. Salsench J, Martínez-Gomis J, Torrent J, Bizar J, Samsó J, Peraire M. Relationship between duration of unilateral masticatory cycles and the type of lateral dental guidance: a preliminary study. Int J Prosthodont. 2005;18:339-346.

23. Mizumori T, Tsubakimoto T, Iwasaki M, Nakamura T. Masticatory laterality-evaluation and influence of food texture. J Oral Rehabil. 2003;30:995-999.

24. Christensen LV, Radue JT. Lateral preference in mastication: a feasibility study. J Oral Rehabil. 1985;12:421-427.

25. Strauss E. Hand, foot, eye and ear preferences and performance on a dichotic listening test. Cortex. 1986;22:475482.

Correspondence: Jordi Martinez-Gomis, Campus de Bellvitge, Universitat de Barcelona, C/Feixa Llarga s/n, 08907 L'Hospitalet de Llobregat, Spain.

E-mail: jmartinezgomis@ub.edu 